mind in the diagnosis of children patients who had complaints that fever, weakness, weight loss and especially joint pain, where pediatric clinics.

\section{P624 HOSPITAL-ACQUIRED NEONATAL MENINGITIS: EXPERIENCE OF THE NEONATOLOGY DEPARTEMET OF SFAX (TUNISIA)}

${ }^{1}$ Amel Ben Hamed, ${ }^{1}$ Adel Babay, ${ }^{1}$ Manel Charfi, ${ }^{2}$ Nour Houda Ben Ayed, ${ }^{1}$ Chiraz Regaieg* ${ }^{1}$ Amira Bouraoui, ${ }^{1}$ Nedia Hmida, ${ }^{2}$ Adnene Hamami, ${ }^{1}$ Afef Ben Thabet, ${ }^{1}$ Abdellatif Gargouri. ${ }^{1}$ Department of Neonatology, Hedi Chaker Hospital, Sfax, Tunisia; ${ }^{2}$ Microbiology Laboratory, Habib Bourguiba Hospital, Sfax, Tunisia

10.1136/archdischild-2019-epa.955

Background The past decade brought with it both highly sophisticated neonatal intensive care with improved perinatal mortality rates and increased risk for nosocomial septicemia and meningitis among survivors. Although most of these infections were caused by multiple antibiotic-resistant bacteria. The absence of specific clinical signs makes diagnosis of meningitis more difficult.

Methods We enrolled neonates who were admitted to neonatal unit at at Hedi Chaker hospital, Sfax from 1 January 2007 to 31 December 2016 and had suspected nosocomial infection with abnormal cerebrospinal fluid examination.

Results Five cases were reviewed, Among these 4 were girls (80\%), All neonates were preterm between 27 and 34 weeks of pregnancy, with Low birth weight in 2 cases and very Low birth weight in 3 cases, the average age of diagnosis was 14.2 days after hospitalization, $4(80 \%)$ of them had concomitant bacteremia, suspected bacterial infection was confirmed in 4 cases $(80 \%)$, by positive results on blood culture in 4 cases but only one cerebrospinal fluid (CSF) culture was positive, Klebsiella pneumoniae was the leading pathogen. The case mortality rate (CMR) was 0.8. Four patients died and one case was cured but he developed a sequellae of psychomotoric retardation.

Conclusion Preterm neonates have a high risk of developing nosocomial infections and especially Nosocomial Meningitis wich is related to a high case mortality rate, Definitive diagnosis is made by cerebrospinal fluid examination (CSF) via lumbar puncture (LP), which should be performed in any neonate suspected of having sepsis or meningitis. Future efforts should be directed toward better definition of bacterial virulence, host susceptibility and preventive measures.

\section{P625 IMPACT OF ANTIBACTERIAL DRUGS' OVERUSE IN DURATION OF HOSPITALIZATION AMONG PATIENTS WITH ACUTE DIARRHEAL DISEASES IN 'NORK' ICH, ARMENIA, 2018}

\footnotetext{
1,2,3 Erik Grigoryan, ${ }^{4}$ Mark Grigoryan, 4,5Vigen Asoyan, 4,5,6 Hripsime Apresyan*. ${ }^{1}$ Armenian State University of Economics, Yerevan, Armenia; ${ }^{2}$ Swiss UMEF University, Yerevan, Armenia; ${ }^{3}$ Amberd Research Center, Yerevan, Armenia; ${ }^{4}$ Yerevan State Medical University after Mkhitar Heratsi, Yerevan, Armenia; ${ }^{5}$ Muratsan University Hospital Complex, Yerevan, Armenia; ${ }^{6}$ Wigmore Clinic, Yerevan, Armenia
}

\subsection{6/archdischild-2019-epa.956}

Background Antibiotics were announced as life-savers when they became widely available in the middle of 20th century. Nowadays, antimicrobials are fast becoming killers themselves. The more any given antibiotic is used, the greater the chances that bacteria will develop antimicrobial resistance (AMR) that renders the drug ineffective.

Although we don't have exact number of deaths from antibiotic-resistant infections in Armenia, we give importance to its prediction and prevention of developing multidrug resistant strains of bacteria. Our goal is to describe the low cost-effectiveness of treatment in accordance with national and international guidelines to hospitalized patients with acute diarrheal diseases. It is an option why not to use antibiotics inappropriate.

Objective The purpose of this study was to evaluate the costeffectiveness of treatment with national guidelines and so to create a premise for prevention of inappropriate antibiotic use.

Methods A retrospective economic analysis was performed using 45-day (01.04.-15.05.2018) data from "Nork" ICH. The analysis was conducted from a third-party payer's perspective.

During the above mentioned period 156 patients were admitted from which $125(80.1 \%)$ had watery and $31(19.9 \%)$ bloody diarrhea. Antibacterial treatment was given to 80 patients, but only in $23(28.75 \%)$ cases it was indicated. Widely used antibacterials were nifuroxazide 55.6\%, azithromycin 4.4\%, amoxicillin 1.1\%, metronidazole 3.3\%, ciprofloxacin 13.3\%, TMP-SMX $6.7 \%$, cefotaxime $1.1 \%$, ceftriaxone $14.5 \%$.

Patients with appropriate antibacterial treatment were hospitalized 6.8 days on average. The patients without any antibacterial treatment and patients with inappropriate antibacterial treatment were hospitalized 4.7 and 6.3 days on average respectively.

Average Total Cost of treatment was calculated as follows:

$\mathrm{a}-$ average duration of hospitalization $b^{*}$ - average cost of treatment per patient per day

* - it is fixed cost - 36.7EUR

Eventually, average costs of treatments have been determined.

Results Since average cost of treatment is known- 36.7EUR per patient per day, totally 172.49EUR per patient, it can be compared with inappropriate treatment cost-totally 231.21EUR per patient. The difference in the costs of two treatment options is 58.72 EUR per patient. Multiplying by the number (57) of patients managed with inappropriate treatment we will have total difference 3347EUR during 45 days.

Conclusions Treatment in accordance with national guidelines is cheaper in comparison with inappropriate antibacterial treatment as the last requires longer duration of hospitalization.

The implementation of guidelines is important from both clinical and financial points of view.

\section{P626 INFLAMMATORY MARKERS OF ANTIPSYCHOTIC WEIGHT GAIN AND CARDIOMETABOLIC DYSFUNCTION IN YOUTH MENTAL HEALTH DISORDERS}

\footnotetext{
1,2Karen Conlan, ${ }^{3,4}$ Eadaoin Hayes*, 'Louise Gallagher, ${ }^{4}$ Nicole Wood, ${ }^{5}$ Andrew Hogan, ${ }^{3}$ Donal O'Shea, 'Jane McGrath. 'Trinity College, Dublin, Ireland; ${ }^{2}$ National Children's Research Centre, Dublin; ${ }^{3}$ University College Dublin, Dublin, Ireland; ${ }^{4}$ National Children's Research Centre, Dublin, Ireland; ${ }^{5}$ NUI Maynooth, Kildare, Ireland
}

\subsection{6/archdischild-2019-epa.957}

Introduction Second generation antipsychotics (SGAs) are prescribed to treat mental health disorders in children. However, there are concerns about these medications due to 